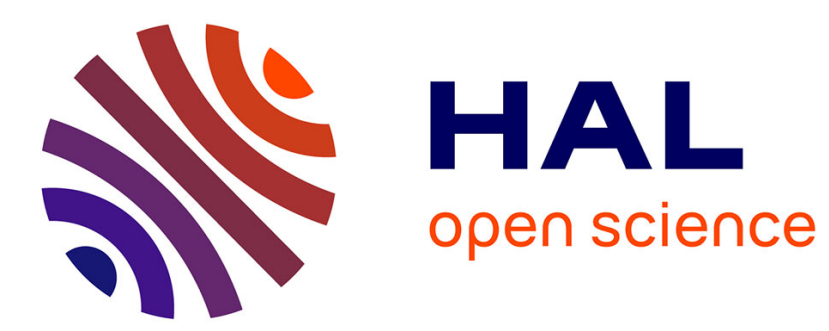

\title{
The trajectories of neutral polarizable particles in an inhomogeneous electric field
}

\author{
J.M. Bermond
}

\section{To cite this version:}

J.M. Bermond. The trajectories of neutral polarizable particles in an inhomogeneous electric field. Revue de Physique Appliquée, 1982, 17 (8), pp.491-498. 10.1051/rphysap:01982001708049100 . jpa00245026

\section{HAL Id: jpa-00245026 https://hal.science/jpa-00245026}

Submitted on 1 Jan 1982

HAL is a multi-disciplinary open access archive for the deposit and dissemination of scientific research documents, whether they are published or not. The documents may come from teaching and research institutions in France or abroad, or from public or private research centers.
L'archive ouverte pluridisciplinaire HAL, est destinée au dépôt et à la diffusion de documents scientifiques de niveau recherche, publiés ou non, émanant des établissements d'enseignement et de recherche français ou étrangers, des laboratoires publics ou privés. 


\title{
The trajectories of neutral polarizable particles in an inhomogeneous electric field
}

\author{
J. M. Bermond \\ C.R.M.C. ${ }^{2}$-C.N.R.S., Campus de Luminy, case 913, 13288 Marseille cedex 09, France
}

(Reçu le 4 février 1982, révisé le 27 avril 1982, accepté le 29 avril 1982)

\begin{abstract}
Résumé. - Les trajectoires de particules neutres polarisables sont déviées par un champ électrique inhomogène. Ceci fournit de nombreuses méthodes pour mesurer la polarisabilité $(\alpha)$ de particules neutres. Parmi celles-ci il semble que la microscopie par émission de champ ait souffert jusqu'à présent d'un manque de précision, malgré ses grandes possibilités. Les propriétés tri-dimensionnelles des trajectoires de particules polarisables sont étudiées dans cet article afin de déterminer leur importance et leur influence sur les méthodes de mesure. On montre d'abord, de façon générale, que l'on peut analyser pratiquement toutes les situations expérimentales en utilisant un paramètre sans dimension $\lambda$ et une loi de similitude. Dans le cas particulier d'un microscope électronique ou ionique de champ on fait un calcul tri-dimensionnel des trajectoires. On trouve de grandes différences avec les résultats des calculs bi-dimensionnels antérieurs. Il faut aussi tenir compte de la distribution des vitesses des particules, le cas échéant. De nouveaux abaques de validité assez générale sont donnés pour la mesure de $\alpha$ avec un microscope électronique de champ. A titre d'exemple, on redétermine la polarisabilité de l'atome de plomb $\left(\alpha=9,7 \pm 2,9 \AA^{3}\right)$.
\end{abstract}

\begin{abstract}
The trajectories of neutral polarizable particles are curved by an inhomogeneous electric field. This provides a variety of methods for measuring the polarizability $(\alpha)$ of neutrals. Amongst these methods, field electron microscopy seems to have been impaired so far by a lack of precision, despite its broad potentialities. The threedimensional properties of the trajectories of polarizable particles are examined in this paper in order to determine their importance and their influence on the measurement methods. It is first shown, as a general property, that by introducing a dimensionless parameter $\lambda$ and by using a scaling law, nearly all experimental situations can be analysed. In the particular case of the field electron or field ion microscope a three-dimensional calculation of the trajectories is made. The results show major differences with those of the previously existing two-dimensional theories. The velocity distribution of the particles is also considered and is found to have a non negligible influence. New graphs of fairly general validity are drawn that can be used to measure $\alpha$ with a field electron microscope. As an example a more correct value of the polarizability of $\mathrm{Pb}$ is determined $\left(\alpha=9.7 \pm 2.9^{\circ} \AA^{3}\right)$.
\end{abstract}

1. Introduction. - The electric dipole polarizability $(\alpha)$ of an atom or a molecule plays an important role in many optical or atomic phenomena [1]. Although theoretical values for $\alpha$ are now available from a variety of calculations, experimental data are either lacking or very imprecise for most of the elements or molecules, particularly those that are not in the gaseous state at normal laboratory temperatures. For this type of particles, a direct method of measurement of $\alpha$ is the deflexion of an atomic or molecular beam by an inhomogeneous electric field [1,2]. This method has been brought to a high accuracy by using either a pure electrostatic deflection [3] or the « E.H. gradient balance " technique [4]. However, its use has been restricted so far to the study of a few elements (mainly alkali and earth-alkali metals) due to $(a)$ the relatively small field gradients (small deviations) which are practically achieved and $(b)$ the lack of an efficient detector for neutral particles. Another type of deflection experiment was introduced many years ago by Drechsler and Müller [5] and later reexamined [6]. In this method the beam is deflected by the highly inhomogeneous field near the tip of a field electron microscope while the tip itself is used as the detector. Very important angular deviations can thus be achieved and measured by analysing the resulting change of the image. Moreover, one can detect in principle any particle which can stick to the surface of the tip and can then be imaged or detected by the microscope itself. This makes this quite simple technique highly efficient for measuring the polarizabilities of a wide variety of neutral particles $[5,7]$.

However, in developing this method, only twodimensional calculations of the particle trajectories 
have been used so far $[5,6]$. The present paper is a general study of the trajectories in three dimensions. Some general properties are first established. Then, it is shown that three-dimensional effects have a major influence on the measured values of $\alpha$, instead of being negligible as previously thought. In the three-dimensional case the distribution of velocities too has to be considered. More specific graphs of fairly general validity for the determination of $\alpha$ by field electron microscopy are presented. As an example the new theory is used to redetermine the polarizability of $\mathrm{Pb}$.

2. General properties of the trajectories. - In this section we assume the polarizability $\alpha$ to be a scalar, unless otherwise stated. We also assume that the initial point $\mathbf{M}_{0}$ of any trajectory lies in a region where the electric field is zero or negligible $\left({ }^{1}\right)$.

2.1 BASIC EQUATIONS. - Let $F_{0}$ be the field at one arbitrary (but conveniently chosen) point in space $\left(^{2}\right.$ ). Then the field at any point $M$ is $F=F_{0} \cdot \gamma(M)$ where $\boldsymbol{\gamma}(\mathrm{M})$ is a vector function of $\mathrm{M}$ which only depends on the geometry of the conductors. The polarization force on the particle is :

$$
\mathbf{f}=\frac{1}{2} \alpha \operatorname{grad} F^{2}=\frac{1}{2} \alpha F_{0}^{2} \operatorname{grad} \gamma^{2} .
$$

Let $\mathbf{v}_{0}$ be the initial velocity of the particle and $\mathbf{v}$ its velocity at $M$. The potential energy of the system is $-\frac{1}{2} \alpha F^{2}$ if we choose the zero of the potential energy at $\mathbf{M}_{0}$. The total energy $W$ of the system is conservative. Then

$$
W=\frac{1}{2} m v_{0}^{2}=\frac{1}{2} m v^{2}-\frac{1}{2} \alpha F^{2}=\frac{1}{2} m v^{2}-\frac{1}{2} \alpha F_{0}^{2} \gamma^{2}
$$

or

$$
v^{2}=v_{0}^{2}\left(1+\lambda \gamma^{2}\right)
$$

where

$$
\lambda=\frac{\alpha F_{0}^{2}}{m v_{0}^{2}} .
$$

Using this definition of $\lambda$, equation (1) may be rewritten

$$
\frac{\mathrm{d} \mathbf{v}}{\mathrm{d} t}=\frac{\lambda v_{0}^{2}}{2} \operatorname{grad} \gamma^{2} .
$$

By taking the curvilinear abscissa $s$ along the trajectory as the independent variable, an easy calculation then yields the differential equation of the trajectory itself

$\frac{\mathrm{d}}{\mathrm{d} s}\left(\left(1+\lambda \gamma^{2}\right) \frac{\mathrm{dr}}{\mathrm{d} s}\right)+\left(1+\lambda \gamma^{2}\right) \frac{\mathrm{d}^{2} \mathbf{r}}{\mathrm{ds} s^{2}}=\operatorname{grad}\left(1+\lambda \gamma^{2}\right)$

${ }^{(1)}$ If this is not the case the general properties of the trajectories are left unaltered, although their differential equation is slightly changed.

$\left({ }^{2}\right) F_{0}$ is not the field at the initial point $M_{0}$. For example if the particle travels in the field of a sphere, $\mathbf{F}_{0}$ may be the field at the surface of this sphere. In the case of a FEM tip, $\mathbf{F}_{0}$ may be the electric field at the apex. where the vector $\mathbf{r}$ describes the position of the particle in some fixed reference frame. Any particular trajectory is. entirely determined by equation (4) and the initial conditions (initial point $\mathbf{M}_{0}$ and initial velocity $\mathbf{v}_{0}$ ).

2.2 Consequences. - Equation (4) has been analytically solved in only two quite simple cases : the central field of a sphere and the field of an infinite cylinder [8]. For more complicated electrode shapes a numerical solution is necessary. Yet, some properties of equation (4) can be used to simplify the study of the trajectories.

(a) It is first seen that for given initial conditions $\left(\mathrm{M}_{0}, \mathbf{v}_{0}\right)$ the trajectory only depends on $\lambda \gamma^{2}$. The dimensionless function $\gamma(\mathbf{r})$ or $\gamma(\mathbf{M})$ is only determined by the geometry of the electrodes. The dimensionless parameter $\lambda$ is determined by the applied potentials (through $F_{0}$ ), the physical properties of the particle (through $\alpha$ ) and its initial kinetic energy $\left(\frac{1}{2} m v_{0}^{2}\right)$. In other words, given the geometry of the electrodes, the initial point, and the orientation of $v_{0}$, the trajectory depends on one and a single parameter $(\lambda)$, which includes all the physical quantities of interest.

(b) A scaling law : if the shape of the electrodes is changed by a similarity of ratio $k$, an arbitrary point $M$ in space is changed into a corresponding point $\mathbf{M}^{\prime}$. If the applied potentials are kept constant in the transformation, the field at $\mathbf{M}^{\prime}$ is that at $\mathbf{M}$ divided by $k$. The field at $\mathrm{M}_{0}^{\prime}$ corresponding to $\mathrm{M}_{0}$ is $F_{0} / k$ too. Thus the functional expression for $\gamma$ in the transformed geometry is the same as in the initial one $\left({ }^{3}\right)$. Equation (4) is thus left unaltered if $\lambda$ is also kept constant. In other words, for a given value of $\lambda$ if the electrodes are transformed by a similarity while the applied potentials are kept constant, the trajectories are transformed by the same similarity. In particular, two corresponding trajectories meet the electrodes at the same corresponding points. Thus, in the case of a parallel beam it is sufficient to study the trajectories as a function of $\lambda$ for one and a single arbitrary size of the electrodes.

2.3 THE MEANING of $\alpha$. - It must be recalled that the polarizability $\alpha$ involved in this calculation is the static polarizability of the particle. This is a scalar

$\left({ }^{3}\right)$ This statement is easily understood in the simple case of a sphere $S$ (centre $O$, radius $R_{0}$ ) at potential $V_{0}$ which is transformed into a sphere $S^{\prime}$ (centre $O$, radius $k R_{0}$ ) at $V_{0}$. The field of $S$ at a point $M$ defined by $O m=r$. u (u unit vector) is $\mathrm{F}=\mathrm{u} \frac{V_{0} R_{0}}{r}$. By taking $F_{0}$ as the field at the surface of $S$ one finds $\gamma(M)=u \cdot \frac{R_{0}^{2}}{r^{2}}$. The field of $S^{\prime}$ at the corresponding point $\mathbf{M}^{\prime}\left(\mathbf{M}^{\prime}=\mathbf{u} k r=u r^{\prime}\right)$ is now $\mathrm{F}^{\prime}=\mathrm{u} \frac{V_{0} R_{0}^{\prime}}{r^{\prime 2}}$ where $R_{0}^{\prime}=k R_{0}$ and $r^{\prime}$ now stands for the new independent space coordinate. $F_{0}$ is now the field at the surface of $\mathrm{S}^{\prime}: \mathbf{F}_{0}=\mathrm{u} \frac{V_{0}}{k R_{0}}=\mathrm{u} \frac{V_{0}}{R_{0}^{\prime}}$. Then $\gamma\left(\mathrm{M}^{\prime}\right)=\mathrm{u} \frac{R_{0}^{\prime 2}}{r^{\prime 2}}$, which is the same functional relationship as $\gamma(\mathbf{M})$. 
quantity in the case of a spherically symmetrical atom only. In general $\alpha$ is a second-rank tensor symmetrical around the axis of symmetry of the particle. For a single particle the quantity which appears in the expression of the deflecting force (eq. (1)) is the tensor component $\alpha_{z z}$ along the direction of the field [1]. It depends on the magnetic quantum number of the atom [1]. In a beam experiment one measures in fact the average polarizability $\alpha$ of the particle, that is the average of $\alpha_{z z}$ over all $m_{J}$. This is the isotropic part of the total polarizability.

3. A polarizable particle in the field of a field emission tip. - 3.1 A THREE-DIMENSIONAL COMPUTATION OF THE TRAJECTORIES. - In this section we are interested in the trajectories of an initially parallel beam of neutral polarizable particles, devoid of any permanent dipole moment, near the tip of a field electron or field ion microscope. The electrodes are the tip itself and the microscope screen. The tip axis is perpendicular to the screen and a revolution symmetry around it is assumed for the whole system.

The particle source is installed so much away from the tip that the beam originates from a practically field-free region.

The radius of curvature at the tip apex is named $R_{0}$. The spacing between the electrodes is characterized by the distance $l$ between the screen and the centre of curvature $\mathbf{O}$ at the tip apex. The interelectrode electric potential is constructed by the known superposition of two weighted potentials with a weighting factor $f$ (form factor) [9]. The field at the tip apex is chosen as the particular value $F_{0}$. By so doing the geometry and the field distribution $(\gamma)$ are completely defined by $R_{0}, l, f$ and $F_{0}$.

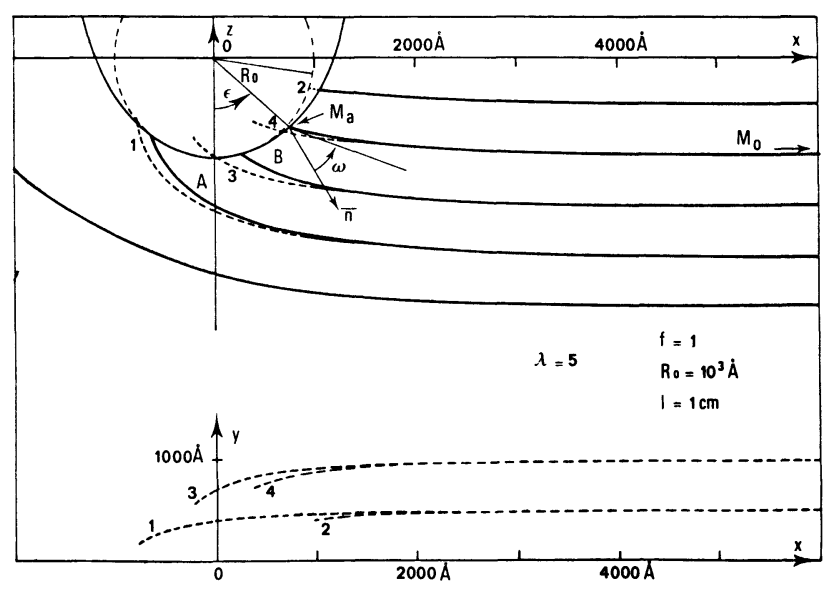

Fig. 1. - Projections of some trajectories on to the symmetry plane of the tip $x \mathrm{O} z$ (top) and on to the $x \mathrm{O} y$ plane, perpendicular to the tip axis (bottom). The tip profile is shown. The particles come from the right with their trajectories initially parallel to Ox. Solid lines : trajectories lying in the symmetry plane. Dashed lines : trajectories situated outside this plane. The two projections of a given curve are both labelled with the same number. $f=0.1$; $R_{0}=10^{4} \AA ; l=1 \mathrm{~cm} ; \lambda=5$.
According to 'vhat was said in section 2.2.a, the problem of calculating one particular trajectory in the incident beam is now totally determined by specifying $R_{0}, l, f, \lambda$, the direction of the initial velocity and the initial point $M$. The following reference frame was chosen : the origin at the centre of curvature of the tip apex. $\mathrm{O} z$ along the tip axis positive towards the shank. $O x$ and $O y$ completing a direct cartesian frame. In particular, the arrival point on the surface $\mathbf{M}_{a}$ is specified by its cartesian coordinates $(x, y, z)$ or by its cylindrical coordinate $(\phi, \varepsilon, z)$. The impingement angle is named $\omega$; it is the angle of the trajectory with the normal to the surface at the arrival point $\mathbf{M}_{\mathbf{a}}$ (see Fig. 1).

For each trajectory, equation (4) was numerically solved using the radius of curvature method. In the particular case of a sphere $(f=1)$ our program could be checked by comparing its results to those of an independent analytical calculation. The coordinates of all points along the trajectory agreed within a few $\AA$ and the angles of impingement on the surface within one tenth of a degree. So we are fairly confident in the present results.

3.2 THE DIVERGENCE OF THE TRAJECTORIES. - As will be seen in section 4 , an important quantity is what we call here the divergence of the trajectories. The meaning of this term is the following : let us consider a trajectory (c) starting from $\mathbf{M}_{0}$ and crossing the tip surface at $\mathbf{M}_{\mathbf{a}}$. An elementary tube of trajectories surrounding (c) has an initial cross-sectional area $\mathrm{d} \sigma$. It has another cross-section $\mathrm{d} \Sigma$ at $\mathbf{M}_{\mathrm{a}}$. The divergence of the trajectories at $M_{a}$ is then $D=\mathrm{d} \Sigma / \mathrm{d} \sigma$ by definition. If $D>1$ the trajectories diverge at $M_{\mathrm{a}}$ whereas they converge if $D<1$. The dimensionless quantity $D$ depends on the "mean " trajectory (c) i.e. on $\lambda$ and the arrival point. $D$ was also computed in our program.

3.3 RESULTS. - Although any orientation of the initial velocity can be taken into account, the results which are presented hereafter are restricted to the case of a particle beam normal to the tip axis. This is an easily achievable and most usual experimental situation.

Figures 1 and 2 show some examples of the computed trajectories. Ox lies along the beam direction $\left(\mathbf{O x} \cdot \mathbf{v}_{0}=-1\right)$. The dimensions of the electrodes are specified by $R_{0}=10^{3} \AA$ and $l=1 \mathrm{~cm}$. The form factor is $f=0.1$ the trajectories lying in the symmetry plane $(x \mathrm{O} z)$ are drawn as solid lines and some of them are labelled with letters; the various projections of those that run outside the symmetry plane are drawn as dashed lines and are labelled with figures.

The influence of $\lambda$ on the shape of the trajectories is seen by comparing figure 1 and figure 2 . Curve 1 on figure 2 shows that a particle leaving the source from one side of the symmetry plane $(x \mathrm{O} z)$ may hit the tip on the other side of this plane. This means that a point on the surface may be hit by particles coming from both sides of the $x \Omega_{7}$ nlane if $\lambda$ is laroe ennugh 


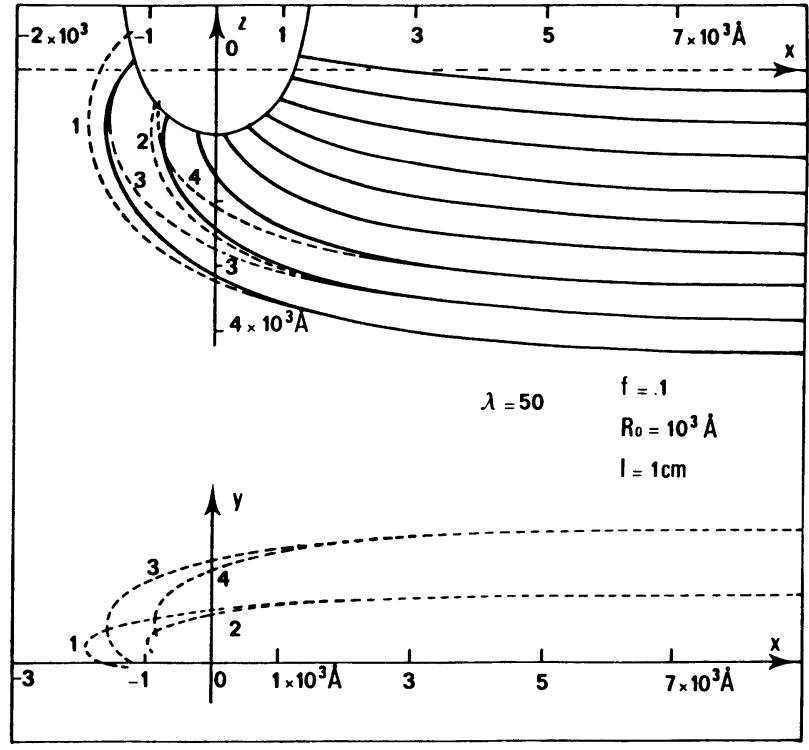

Fig. 2. - Same type of projections as in figure 1 (with a different scale) for $\lambda=50$ and $f, R_{0}, l$ the same as for figure 1 . The "focussing" properties of the tip are clearly visible.

Moreover, curves 1 and 3 suggest that the tip has some "focussing" properties i.e. a caustic surface exists, which leads to an accumulation of the trajectories in its vicinity. However an investigation of the curve shapes showed that both of these phenomena only occur outside the visible portion of the tip $\left(\varepsilon \leqslant 45^{\circ}\right)$ for all values of $\lambda$ of experimental interest $(\lambda \leqslant 50)$. Therefore no further effort was made to precisely determine a caustic surface.

Figure 1 also shows why a two-dimensional calculation is misleading for determining the divergence of the trajectories. Curves (A) and (B) which lie in the symmetry plane $x \mathrm{O} z$ do diverge in the vicinity of the tip. However the value of $D$ at $A$ is 0.605 , i.e. all the neighbouring trajectories outside the plane converge around $A$. This effect was misevaluated in our previous article [6] and its importance must be stressed here.

Our results can be presented in various manners, each of which refers to a specific method for determining $\lambda$ and subsequently $\alpha$. A mapping of the points of impingement on the surface is useful if one is able to detect individual particles deposited upon the tip (such as in field ion microscopy). Such a map is shown in figure $3(\lambda=5)$. The various trajectories start from an array of regularly spaced initial points. The figure is a projection of the impingement points on to the $x \mathrm{O} y$ plane (small solid dots). It again displays the importance of three-dimensional effects : for example the particles which start from $y_{0}=0.15 R_{0}$ at various $z_{0}$ hit the tip at an ordinate of $0.1 R_{0}$ or less. With no field the shadowed region of the tip is on the left side of the $\mathrm{O} y$ axis. With the field applied some particles reach the shadow region. From an experimental measurement of their number the value of $\lambda$ can be determined, which leads to the determination of $\alpha$ once $F_{0}, m$ and $v_{0}$ are known. In this paper we shall

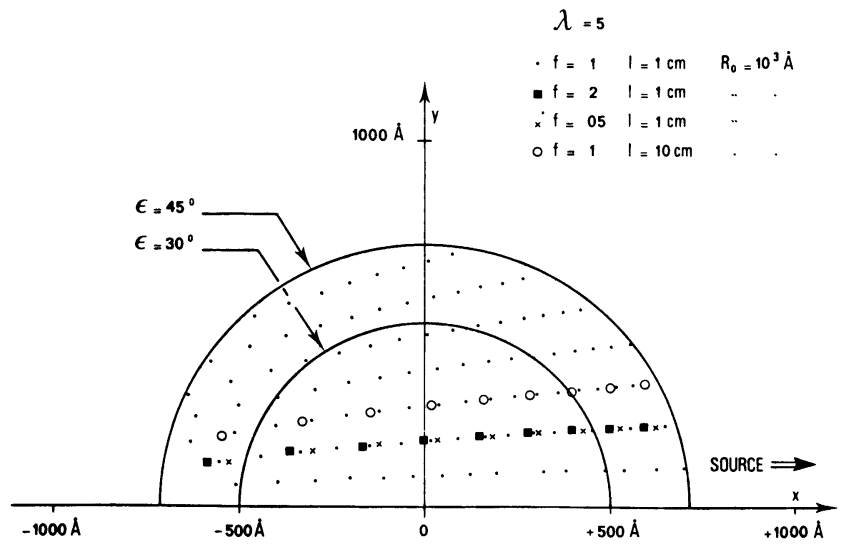

Fig. 3. - Density of the impingement points over one half of the usable tip surface in the particular case of an homogeneous beam of particles perpendicular to the tip axis. The beam comes from the right with the trajectories initially parallel to $O x$. The trajectories start from a regular array of points $\left(\Delta y_{0}=\Delta z_{0}=0.15 R_{0}\right)$. The impingement points are seen in projection on to the plane of the figure perpendicular to the tip axis (projected at $\mathrm{O}$ ). Only those points are shown that correspond to $\varepsilon \leqslant 45^{\circ}$ (outer circle). The practically usable portion of the tip is limited by the inner circle $\left(\varepsilon=30^{\circ}\right)$. The points lying in the symmetry plane (i.e. along $O x$ ) have been omitted. $\lambda=5$. Small dots : impingement points for $f=0.1, R_{0} / l=10^{-5}$. The density decreases from the right to the left. However, the influence of the convergence is noticeable on going from the centre to the rim of the circles. The lines along which the points apparently fall are no projections of the trajectories. Influence of the parameters $f$ and $R_{0} / l$ (Squares ( $\square$ ), crosses ( $x$ ) and circles $(O))$ : The trajectories start from the same array of points. Only a few impingement points are plotted for clarity. Squares $(\boldsymbol{\square})$ and crosses $(x)$ : influence of $f ; y_{0}=0.3 R_{0}$ and $\Delta z_{0}=0.3 R_{0}$. Circles (O) : influence of $R_{0} / l ; y_{0}=0.45 R_{0}$ and $\Delta z_{0}=0.3 R_{0}$.

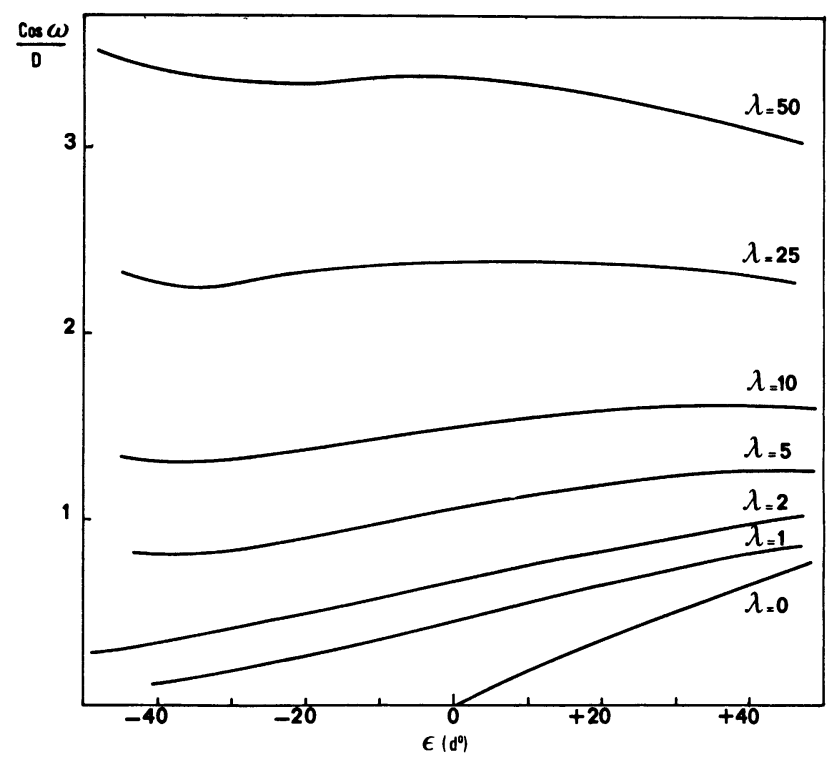

Fig. 4. $-\cos \omega / D$ versus $\varepsilon$ for different $\lambda$ values $(f=0.1$; $\left.R_{0}=10^{3} \AA ; l=1 \mathrm{~cm}\right)$. 
not say more about this method which is currently under investigation. In field electron microscopy, individual particles are not visible on the tip surface whereas the local degree of coverage may be readily measured. Then, an important parameter for the experimental determination of $\lambda$ is $\cos \omega / D$ (see section 4.1). Figure 4 is a plot of $\cos \omega / D$ versus $\varepsilon$ with $\lambda$ as a parameter for the particular tip profile which lies in the symmetry plane $x \mathrm{O} y(\phi=0)$. The shadow region corresponds to $\varepsilon<0$. For small values of $\lambda, \cos \omega / D$ decreases with decreasing $\varepsilon$. For larger values of $\lambda, \cos \omega / D$ goes through a maximum and may even increase with decreasing $\varepsilon$. In other words the local density of impinging particles may be greater on the back of the tip than on its front. This curious effect is entirely due to the decrease of $D$ with $\varepsilon$ and again points to the importance of the convergence factor $D$.

3.4 DiscusSION OF THE THEORETICAL RESUlTS. (a) The following preliminary remarks will be useful in the discussion of our results. First, the visible portion of the tip in a field electron or field ion microscope is practically limited to $\varepsilon \leqslant 45^{\circ}$. Then, the linear dimensions of this useful portion are extremely small with respect to the diameter of the particle beam. This will allow us to consider the beam as infinitely broad in the following sections. Finally, the achievable order of magnitude for $\lambda$ must be kept in mind : assuming a Knudsen velocity distribution in the beam, a reasonable value for $\lambda$ is that corresponding to the most probable velocity, i.e. $\lambda_{\mathrm{m}}=\frac{\alpha F_{0}^{2}}{3 k T}$, where $T$ is the source temperature and $k$ the Boltzmann's constant. $F_{0}$ must not exceed the free-space ionization field, roughly $5 \times 10^{7} \mathrm{~V} / \mathrm{cm}$. For a particule of polarizability $\alpha=10 \AA^{3}, \lambda_{\mathrm{m}}$ would then be 0.84 for $T=800 \mathrm{~K}$ or 0.27 for $T=2500 \mathrm{~K}$. Taking into account the velocity distribution would not involve values of $\lambda$ greater than $50 \lambda_{\mathrm{m}}$ (see section 4), i.e. 42 . and 13.5 respectively in the present example. So, we can restrict our interest to values of $\lambda$ in the range $0 ; 50$. By so doing we exclude the problem of the gas supply function in a field ion microscope, which is beyond the scope of this paper.

(b) The influence of $l, R_{0}$ and $f$ on our results will now be discussed. As $l$ and $R_{0}$ are only involved through their ratio (section 2.2.b), we are left with only two parameters $: R_{0} / l$ and $f$. The influence of $R_{0} / l$ can be seen in figure 3. The small solid dots and the white circles show the arrival points of particles leaving from the same initial points, respectively for $R / l=10^{-5}$ and $10^{-6}$. They do not hit the tip at the same places. Conversely a point of the surface is reached by particles coming from different initial positions when $R_{0} / l$ is varied. However, the total number of particles impinging on the visible region is left unaltered as well as their local density. As the impingement angle $\omega$ may also vary with $R_{0} / l, \cos \omega / D$ was calculated for

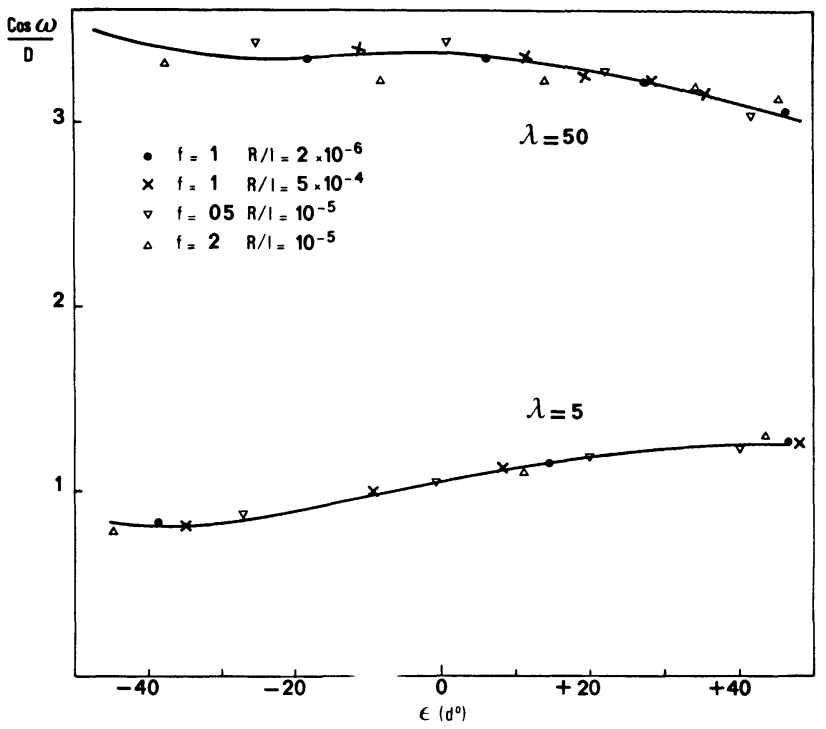

Fig. 5. - Influence of $f$ and $R_{0} / l$ on $\cos \omega / D$ for $\lambda=5$ and $\lambda=50$.

values of $R_{0} / l$ ranging from $2 \times 10^{-6}$ to $5 \times 10^{-4}$. The results are shown on figure 5. All the points practically fall along the same curve. So, a precise experimental measurement of $R_{0} / l$ is not really critical for the determination of $\lambda$, then of $\alpha$.

The influence of the form factor $f$ was also examined within the range of values that are found in practice for field emission tips $(0.05 \leqslant f \leqslant 0.2)$. A comparison of the arrival points for $f=0.05,0.1$ and 0.2 is shown on figure 3. The same conclusion can be drawn about the number and the density of the points. Figure 5 also shows $\cos \omega / D$ versus $\varepsilon$ for the same values of $f$. Although different curves are found, the relative error on $\cos \omega / D$ is less than $4 \%$ for $\lambda=5$ and $6 \%$ for $\lambda=50$ over the usable portion of the tip. This rather small error will even be weighted out by the velocity distribution (see section 4). In conclusion, we consider our present results as valid for any electrode shape that can be practically realized.

(c) Amongst the various representations of the electrostatic potential of the tip $[9,10]$ the superposition of Drechsler and Henkel was chosen because of its analytical simplicity. Whether it yields a realistic description of the field will now be briefly discussed. We shall first point out that the field distribution in the vicinity of the tip seems to be the major parameter. This is supported by merely looking at the deviation of the trajectories which mainly occurs within ten radii of curvature from the tip apex (Fig. 1 and Fig. 2). Now, our method is equivalent to more complicated ones for the representation of the potential and field in the vicinity of the tip [11]. Yet it has been shown [12] that changes in the geometry of the other electrodes (walls, screen) can induce non negligible changes in the field near the tip without altering the equipotential surfaces very much. This may influence the trajectories. However, for a determination of $\lambda$ (or $\alpha$ ) 
we are interested either in the number of particles that hit the tip or in $\cos \omega / D$. As these quantities are practically insensitive to $R_{0} / l$ and $f$ we think our representation of the tip potential is reasonably adequate to our purpose.

4. The measurement of $\alpha$ with a field electron microscope. Application. - 4.1 PRINCIPLE. - In the particular case of a field electron microscope, the local density of deposited particles (or the local coverage) can be measured at some selected point of the tip surface, for example by means of a probe hole. The polarizability of the particles can then be determined from a measurement of the densities $n_{F}$ and $n_{0}$ of deposited particles respectively with and without field [6]. This method is reexamined in this section.

We first assume that a monokinetic beam of particles leaves the initial plane with a density $j_{0}$, and we calculate the number of particles that impinge on an area $\mathrm{d} S$ of the tip per unit time. They come from an area $\mathrm{d} \sigma$ of the initial plane. Without field their density on the tip is

$$
n_{0}=\frac{j \cdot \mathrm{d} \sigma}{\mathrm{d} S / \cos \beta}=\cos \beta \cdot j_{0} \text { per unit time, }
$$

where $\beta$ is the angle of the normal to the tip with the direction of the beam. With the field applied they now hit the surface under the angle $\omega$ with the normal and the cross-section of the tube of trajectories is now $\mathrm{d} \Sigma$ at the impingement point. Then :

$$
\begin{aligned}
& n_{\mathrm{F}}=\frac{j_{0} \mathrm{~d} \sigma}{\frac{\mathrm{d} \Sigma}{\cos \omega}}=\frac{\cos \omega}{D} j_{0} \\
& \frac{n_{\mathrm{F}}}{n_{0}}=\frac{\cos \omega}{D} \frac{1}{\cos \beta} .
\end{aligned}
$$

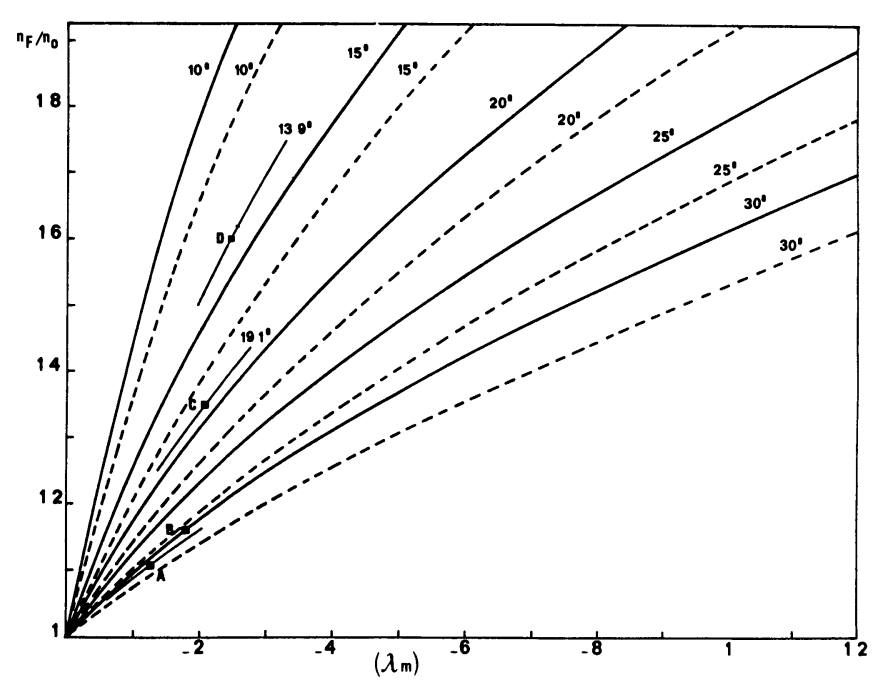

Fig. 6. $-n_{\mathrm{F}} / n_{0}$ versus $\lambda$ or $\lambda_{\mathrm{m}}$. Dashed lines : $n_{\mathrm{F}} / n_{0}$ as a function of $\lambda$ without considering the velocity distribution. Solid lines : $n_{\mathrm{F}} / n_{0}$ as a function of $\lambda_{\mathrm{m}}$ with consideration of the velocity distribution. A, B, C, D : experimental points for $\mathrm{Pb}$. Each curve is relative to one impingement point along the profile $(\phi=0)$ and is labelled with the corresponding value of $\varepsilon$.
This ratio is a function of the impingement point ( $\varepsilon$ and $\phi$ ) and of $\lambda$. Figure 6 shows $n_{\mathrm{F}} / n_{0}$ versus $\lambda$ (dotted lines) for several point (several $\varepsilon$ ) along the tip profile $(\phi=0)$. Only positive values of $\varepsilon$ have to be considered in this method. If the ratio $n_{F} / n_{0}$ has been experimentally determined at a selected point on the profile, the graphs of figure 6 yield the value of $\lambda$ for the particle. Once $F_{0}, v_{0}$ and $m$ are known the polarizability $\alpha$ can be calculated from the definition of $\lambda$ (eq. 3).

In equation (5) $\cos \beta$ also depends on the form factor $f$. However, its variation is less than $\pm 2 \%$ for $\varepsilon=30^{\circ}$ and less than $\pm 1 \%$ for $\varepsilon=20^{\circ}$. Additionally this systematic variation partially compensates for the influence of $f$ on $\cos \omega / D$. Thus we consider the graphs of figure 6 to be valid for any $f$ in the range $[0.05 ; 0.2]$ provided we restrict ourselves to $\varepsilon \leqslant 30^{\circ}$.

\subsection{INFLUENCE OF THE VELOCITY DISTRIBUTION. -} The use of velocity selector has been restricted so far to a few high precision measurements [1]. Considering the simplicity and the wide range of application of experiments using a field emission microscope it seems worthwhile to discuss the influence of the distribution of velocities in the beam. If the beam is not monokinetic the particles have different $\lambda$ depending on their initial velocity $v_{0}$. Then, a point on the tip surface is reached by particles of various $\lambda$ that come from different initial points.

Assuming a maxwellian velocity distribution inside the particle source, the number of particles crossing an elementary surface $\mathrm{d} \sigma$ per unit time in the initial plane with a velocity range from $v$ to $v+\mathrm{d} v$ is

$$
N(v) \mathrm{d} v=A\left(\frac{m}{2 k T}\right)^{3 / 2} \exp -\frac{m v^{2}}{2 k T} v^{3} \mathrm{~d} v \mathrm{~d} \sigma
$$

where $A$ is a proportionality constant. The most probable velocity is $v_{\mathrm{m}}=\left(\frac{3 k T}{m}\right)^{1 / 2}$ which corresponds to $\lambda=\lambda_{\mathrm{m}} \equiv \frac{\alpha F_{0}^{2}}{3 k T}$. finds

Using the dimensionless variable $x=\lambda / \lambda_{\mathrm{m}}$ one

$$
n(x) \mathrm{d} x=A \mathrm{~d} \sigma \frac{9}{2^{5 / 2} \pi^{3 / 2}}\left(\frac{k T}{m}\right)^{1 / 2} \psi(x) \mathrm{d} x
$$

where

$$
\psi(x)=\exp \left(-\frac{3}{2} \frac{1}{x}\right) x^{-3}
$$

The graph of $\psi(x)$ is plotted on figure 7 for $0 \leqslant x \leqslant 5$. $\psi(x)$ reaches its maximum at $x=0.5\left(\lambda=\lambda_{\mathrm{m}} / 2\right)$. $\psi(x)$ is vanishingly small for $x \geqslant 10$. As $\lambda_{\mathrm{m}}$ is always small (section 3.4.a) the particles having a high $x$ (a high $\lambda$ ) only contribute a correction term to the total number of atoms reaching the tip. This makes the influence of $f$ quite negligible in the determination of $\lambda$ (section 3.4.a). Nevertheless the rapid increase 


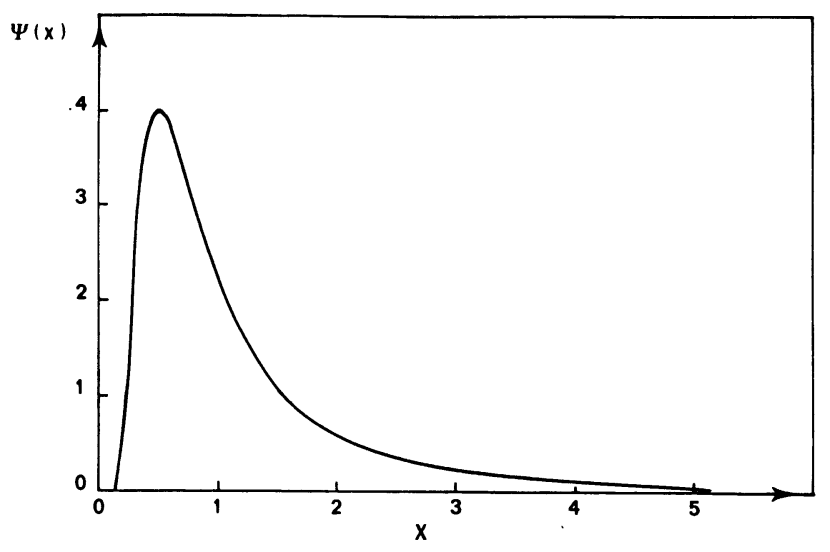

Fig. 7. $-\psi(x)$ as a function of $x$ for $0 \leqslant x \leqslant 5$.

of $\cos \omega / D$ with $\lambda$ makes it necessary to consider the influence of the velocity distribution on the graphs of figure 6 . With the field applied, the density of particles which are deposited around a point of the tip per unit time now becomes :

$$
\begin{aligned}
& n_{\mathrm{F}}=\int_{0}^{\infty} \frac{n(x) \mathrm{d} x}{\mathrm{~d} S}=A \frac{9}{2^{5 / 2} \pi^{3 / 2}}\left(\frac{k T}{m}\right)^{1 / 2} \times \\
& \times \int_{0}^{\infty} \frac{\cos \omega}{D} \psi(x) \mathrm{d} x
\end{aligned}
$$

with the same notations as in section 4.1. Without field this density is

$$
\begin{aligned}
n_{0}=A \frac{9}{2^{5 / 2} \pi^{3 / 2}}\left(\frac{k T}{m}\right)^{1 / 2} \int_{0}^{\infty} \psi(x) \mathrm{d} x \cos \beta & = \\
& =A \frac{9}{2^{5 / 2} \pi^{3 / 2}}\left(\frac{k T}{m}\right)^{1 / 2} \frac{4}{9} \cos \beta .
\end{aligned}
$$

Then,

$$
\frac{n_{\mathrm{F}}}{n_{0}}=\frac{1}{\cos \beta} \frac{9}{4} \int_{0}^{\infty} \frac{\cos \omega}{D} \psi(x) \mathrm{d} x .
$$

As $x=\lambda / \lambda_{\mathrm{m}}$ this ratio is now a function of $\lambda_{\mathrm{m}}$ and $\varepsilon$ and $\phi$. The integral in (7) has been numerically calculated for the same tip profile as in section 3.5 $(\phi=0)$. The results are also shown on figure 6. A given value of $n_{\mathrm{F}} / n_{0}$ is now obtained for a $\lambda_{\mathrm{m}}$ smaller by $20 \%$ or $25 \%$. So, the distribution of velocities in the beam has to be considered in any cases. Its influence was thought negligible in the previous papers $[5,6]$ which only relied on two-dimensional calculations. The present example makes plain the importance of three-dimensional effects.

4.3 Application : the POlaRizability of LeAD ATOMS. - A more correct value of the polarizability of the $\mathrm{Pb}$ atom can be determined by using the new graphs of figure 6. In our previous paper [6] we had measured the times $\tau_{n}$ and $\tau$ that are needed to reach a given coverage on some single crystal faces of the tip, respectively without and with an applied field. Then the ratio $\tau_{0} / \tau$ is equal to $n_{\mathrm{F}} / n_{0}$, assuming an equal sticking coefficient in both cases. The experimental points are plotted in figure 6 . As the ratios $\tau_{0} / \tau$ were measured with an uncertainty of 3 to $4 \%$, all the corresponding $\lambda_{\mathrm{m}}$ fall within the same domain of error. Their average value is then $\lambda_{\mathrm{m}}=0.192$. However, if we discard point $\mathrm{A}$ whose position may be too sensitive to an experimental error on the form factor $f$, we find an average $\lambda_{\mathrm{m}}=0.213$. From the measured values of $F_{0}, v_{0}$ and $m\left(F_{0}=0.32 \mathrm{~V} / \AA, v_{0}=317 \mathrm{~m} / \mathrm{s}\right.$, $m=207$ ), one then calculates the polarizability of the lead atom : $\alpha=9.7 \AA^{3}$. The uncertainty that may affect our determination of $\alpha$ has been discussed in the relevant paper [6]. We just recall here that it is due to the experimental errors on $v_{0}, \tau_{0}, \tau$ and $F_{0}$ with the major contribution $(20 \%)$ coming from $F_{0}$ (although this later error may conceivably be reduced in the future). The relative uncertainty has been evaluated as $30 \%$. Thus $\alpha=9.7 \pm 2.9 \AA^{3}$ for the $\mathrm{Pb}$ atom according to our present determination. We would point out that the present result is half our previous value $\left(\alpha=19.5 \pm 6 \AA^{3}\right)$, although the same experimental data are used. This again points to the importance of three-dimensional effects in such a measurement.

No other experimental value of $\alpha$ for the $\mathrm{Pb}$ atom is available at the moment for a comparison. Theoretical calculations by Thorhallson et al. [13] and by Fraga et al. [14] yield $6.28 \AA^{3}$ and $11.7 \AA^{3}$ respectively. These theoretical values seem quite sensitive to the choice of a basic set of wave functions. However, we consider they are in better agreement with the present experimental value than with our previous one. Yet, it might be noticeable that we found here a polarizability much smaller than the atomic volume of the atom $\left(22 \AA^{3}\right)$.

5. Conclusion. - In this paper we have shown how the study of the trajectories of polarizable particles in an inhomogeneous electric field can be simplified by using the dimensionless parameter $\lambda$ and the scaling law of section 2.2. In the particular case of the field electron or field ion microscope, this has allowed us to construct graphs which can be used in nearly all experimental arrangements to determine the polarizability of a particle. The importance of the convergence of the trajectories (in three-dimensions) is also stressed in this paper, an effect which has not been taken into account by the previous two-dimensional calculations. As a consequence the corrected value of the polarizability of $\mathrm{Pb}$ is about half that previously determined. In this paper, most of the graphs have been constructed with a view to analysing field electron emission data. The application of the theory to the 
specific case of field ion microscopy is left to a future paper.

Acknowledgments. - We thank Dr. M. Drechsler, Directeur de Recherches au C.N.R.S., for his constant interest in the development of this work. The competence and friendship of Dr. J. Bardon, Dr. M. Audiffren and Dr. R. Morin, never faltered throughout many lively discussions, for which it is gratefully acknowledged.

\section{References}

[1] Miller, T. M. and Bederson, B., Advances in Atomic and Molecular Physics, D. R. Bates and B. Bederson eds. (Acad. Press N.Y.) 13 (1977) 1.

[2] Scheffers, H., Phys. Z. 35 (1934) 425.

[3] Schwartz, H. L., Miller, T. M. and Bederson, B., Phys. Rev. A 10 (1974) 1924.

[4] Salop, A., Pollack, E. and Bederson, B., Phys. Rev. 124 (1961) 1431.

[5] DrechsleR, M. and Muller, E. W. Z., Phys. 132 (1952) 195.

[6] Brosse, J. B., Bermond, J. M. and Drechsler, M., Revue Phys. Appl. 11 (1976) 677.

[7] LiEPACK, H. and DreCHSLER, M., Naturwiss. 3 (1956) 52.
[8] Muller, E. W. and Tsong, T. T., Field Ion Microscopy (Elsevier N.Y.) 1969, p. 23.

[9] Drechsler, M. and Henkel, E., Ż. Angew. Phys. 6 (1954) 341.

[10] Dyke, W. E., Trolan, J. K., Dolan, W. W. and Barnes, G., J. Appl. Phys. 24 (1953) 570.

[11] Dyke, W. P. and Dolan, W. W., Adv. Electron. 8 (1956) 89.

[12] Gipson, G. S., Yannitell, D. N. and Eaton, H. C., J. Phys. D 12 (1979) 987.

[13] Thorhallson, J., Fisk, C. and Fraga, 'S., J. Chem. Phys. 49 (1968) 1987.

[14] Fraga, S., Karwowski, J. and Saxena, K. M. S., At. Data Nucl. Data Tables 12 (1973) 467. 